\title{
Gracilis pull-through flap for the repair of a recalcitrant recto-vaginal fistula
}

\author{
Wan Loong James Mok ${ }^{1}$, Ming Hui Goh ${ }^{2}$, Choong Leong Tang ${ }^{2}$, Bien Keem Tan ${ }^{1}$ \\ Departments of ${ }^{1}$ Plastic, Reconstructive and Aesthetic Surgery and ${ }^{2}$ Colorectal Surgery, Singapore General Hospital, Singapore
}

\begin{abstract}
Recto-vaginal fistulas are difficult to treat due to their high recurrence rate. Currently, no single surgical intervention is universally regarded as the best treatment option for rectovaginal fistulas. We present a case of recurrent recto-vaginal fistula surgically treated with a gracilis pull-through flap. The surgical goals in this patient were complete excision of the recto-vaginal fistula and introduction of fresh, vascularized muscle to seal the fistula. A defunctioning colostomy was performed 1 month prior to the present procedure. The gracilis muscle and tendon were mobilized, pulled through the freshened recto-vaginal fistula, passed through the anus, and anchored externally. Excess muscle and tendon were trimmed 1 week after the procedure. Follow-up at 4 weeks demonstrated complete mucosal coverage over an intact gracilis muscle, and no leakage. At 8 weeks post-procedure, the patient resumed sexual intercourse with no dyspareunia. At 6 months post-procedure, her stoma was closed. The patient reported transient fecal staining of her vagina after stoma reversal, which resolved with conservative treatment. The fistula had not recurred at 20 months post-procedure. The gracilis pull-through flap is a reliable technique for a scarred vagina with an attenuated rectovaginal septum. It can function as a well-vascularized tissue plug to promote healing.
\end{abstract}

Keywords Rectovaginal fistula / Colorectal surgery / Reconstructive surgical procedures
Correspondence: Bien Keem Tan Department of Plastic, Reconstructive and Aesthetic Surgery, Singapore General Hospital, Outram Road, Singapore 169608, Singapore Tel: $+65-6-3214686$ Fax: +65-6-2273573 E-mail: bienkeem@gmail.com

Received: 2 May $2018 \bullet$ Revised: 8 Oct $2018 \bullet$ Accepted: 22 Oct 2018

pISSN: 2234-6163・ elSSN: 2234-6171・https://doi.org/10.5999/aps.2018.00416• Arch Plast Surg 2019;46:277-281

\section{INTRODUCTION}

A recto-vaginal fistula is an abnormal epithelium-lined tract between the rectum and vagina, giving rise to symptoms of flatus and fecal or purulent discharge from the vagina. Recto-vaginal fistulas are most frequently caused by trauma [1], but can also be caused by Crohn's disease, pelvic surgery, malignancy, and radiation therapy. Recto-vaginal fistulas are classified according their location. Low fistulas are around the introitus, middle fistulas are between the cervix and the introitus, and high fistulas are close to the cervix [2].

The current management of recto-vaginal fistulas depends on the fistula level. Low and middle fistulas are amenable to transvaginal or perineal approaches allowing closure with a fistula plug [3], fibrin glue [4], local flap [5], interposed tissue [6], or mesh [7]. High fistulas require rectal resection and coloanal anastomosis [8].

Up to $80 \%$ of recto-vaginal fistulas recur after treatment [9]. Fistulas recur due to infection, chronic inflammation, pressure necrosis, repeated trauma, previous radiation therapy, and comorbidities such as diabetes, hypertension, and smoking. Multiple surgical attempts at treatment result in scarring and thinning of the recto-vaginal septum, increasing the risk of recurrence. There is currently no single surgical intervention that is univer- 
sally regarded to be the best option for the treatment of rectovaginal fistulas.

We present a case of recurrent recto-vaginal fistula surgically treated with a gracilis pull-through flap.

\section{CASE}

The patient's history is presented below. Informed consent was obtained.

The patient was a 32-year-old who had a normal vaginal delivery and presented with fecal discharge from the vagina 1 year later. She was diagnosed as having low recto-vaginal fistula and an anterior sphincter direct repair was performed. However, it was complicated by wound infection, which required seton drainage and a temporary diverting colostomy. A repeat sphincter repair and Martius flap were attempted. Recurrence of the recto-vaginal fistula was discovered and anterior levatorplasty with secondary closure was performed. The patient suffered a second recurrence and a left gracilis interposition flap was performed. Thereafter, a third recurrence developed (Fig. 1). For a more secure reconstruction in view of previous failures, reconstruction with a gracilis pull-through flap was planned by the present reconstructive team.

The surgical goals for this patient were complete excision of the fistula tract, thorough debridement of all infected tissue, and introduction of fresh, vascularized muscle to plug the defect. The fistula was delineated and completely excised, resulting in a $7 \times 7 \mathrm{~mm}$ fresh communication between the vagina and rectum. As the recto-vaginal septum was thin, it was not feasible to separate the components for closure.

A gracilis muscle flap, including its tendon, was elevated using

\section{Fig. 1. Recto-vaginal fistula tract}

The tract is shown by the fistula probe passed through the vagina and exiting at the anus.

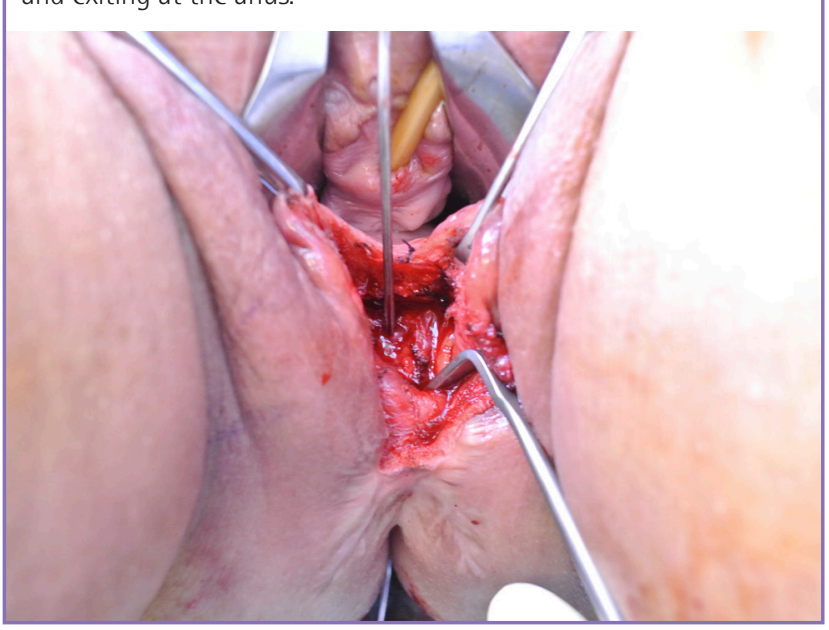

a medial thigh incision, with a supplementary incision over the medial aspect of the tibial tuberosity. Flap dissection was performed in the usual fashion, with care taken to safeguard the great saphenous vein proximally and the saphenous nerve distally. The gracilis was traced down to its insertion over the medial aspect of the tibial tuberosity. The gracilis tendon was disinserted, taking care to preserve the sartorius and semitendinosus insertions. The tendon was necessary to perform the pullthrough. The gracilis flap was then tunneled upwards and passed through the subcutaneous plane to emerge at the introitus. The vaginal mucosa was incised to enlarge the passage for the gracilis. Vicryl (Ethicon Inc., Somerville, NJ, USA) 2/0 sutures were used to secure the muscle to the adjoining vaginal mucosa. The mucosal wound was left open to avoid undue pressure on the flap, allowing for healing via secondary intention. The gracilis tendon was passed through the anus and anchored externally on the skin using Prolene (Ethicon Inc.) 2/0 sutures (Fig. 2).

One week later, the patient underwent a follow-up examination under anesthesia. This examination was performed in the prone jackknife position to allow direct visualization of the anterior rectal mucosa and the gracilis tendon (Fig. 3). Vicryl 2/0 sutures were placed to repair the mucosa, taking a bite of the gracilis to prevent muscle retraction. The excess muscle and tendon were trimmed to be flush with the rectal mucosa (Fig. 4). Postoperatively, the vaginal wound bed was irrigated daily with normal saline till mucosal healing was complete.

Follow-up at 4 weeks demonstrated complete mucosal coverage over an intact gracilis muscle, and no leakage. This was verified under direct vision and on digital rectal examination. At 8 weeks after the procedure, the patient resumed sexual inter-

\section{Fig. 2. Gracilis tendon pull-through}

The gracilis tendon was pulled through the defect and secured outside the anus.

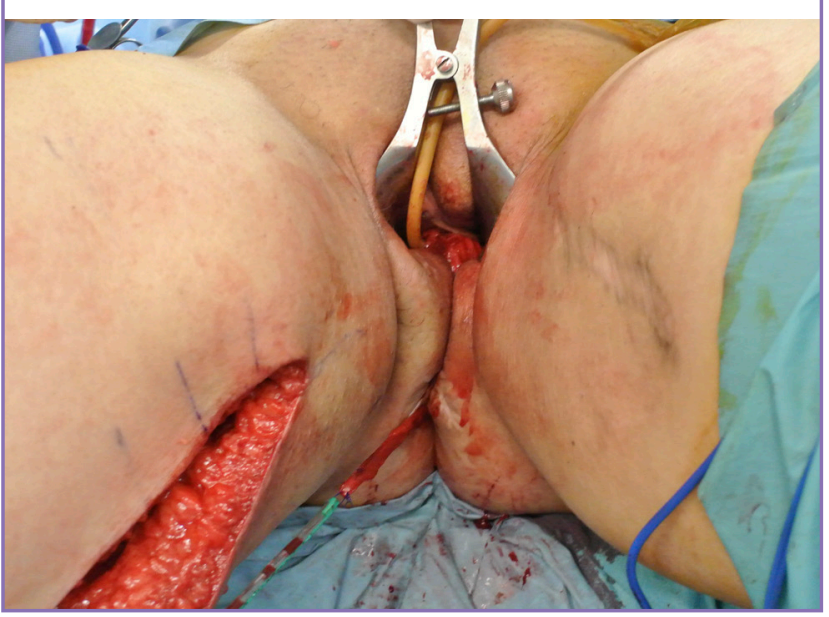




\section{Fig. 3. Endoscopic view of the anterior rectal wall}

The gracilis tendon shown here is held in place by a skin suture and surrounded by healthy rectal mucosa.

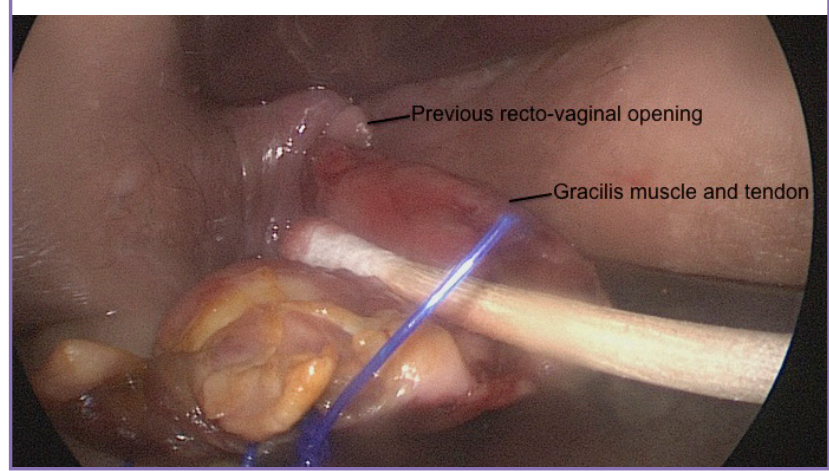

Fig. 4. Schematic overview of the gracilis flap

The excess muscle and tendon were trimmed off the gracilis pullthrough flap 1 week after the procedure.

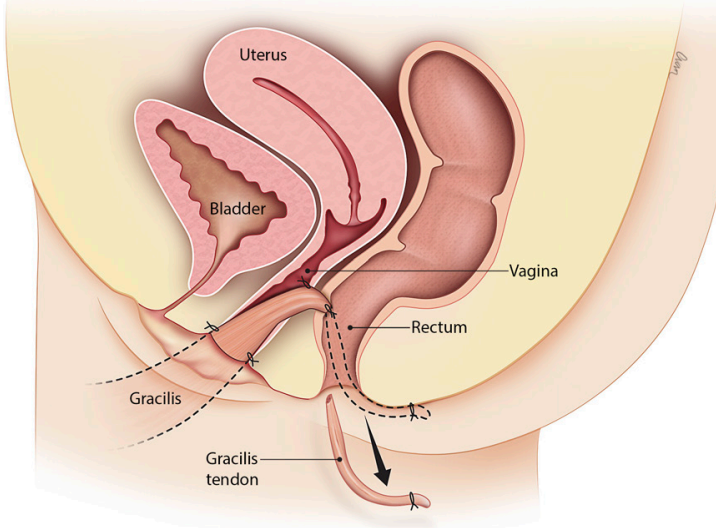

course with no dyspareunia or wound problems. At 6 months after procedure, her stoma was closed. One week after stoma reversal, the patient experienced fecal leakage into the vagina. The problem was rectified after the patient underwent biofeedback for sphincter muscle retraining. In addition, she was taught to perform gentle manual evacuation to aid voiding. The transient leakage lasted 1 month. There was no further leakage or fistula recurrence at 20 months post-procedure (Fig. 5). Manual palpation of the recto-vaginal septum elicited a referred sensation to the medial thigh, indicating that the gracilis was intact.

\section{DISCUSSION}

The gracilis muscle has previously been used for tissue interposition within the recto-vaginal septum, with success rates ranging from $50 \%$ to $90 \%[2,6,10]$. In this case, the previous left gracilis flap was found to be viable but retracted, which caused
Fig. 5. Healed vagina 20 months after the procedure

The gracilis muscle had completely mucosalized and was integrated well into the vaginal wall.

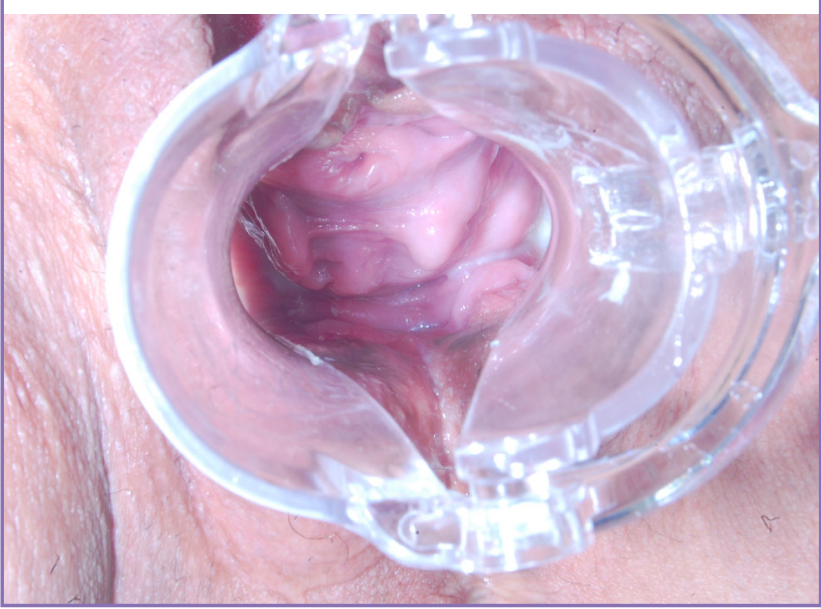

recurrence. The present gracilis design included its tendon to allow a pull-through for added security. The standard gracilis harvest procedure has minimal donor site morbidity, consisting of a medial thigh scar and a short-term decrease in leg function [11]. In this extended harvest, however, the patient experienced more discomfort due to dissection over the knee joint. Care was taken to preserve the sartorius and semitendinosus tendons, as they run adjacent to the gracilis tendon, forming the pes anserinus. It is known that injury to the pes anserinus can cause chronic medial knee pain. Additionally, the saphenous nerve passes superficial to the tendons, meaning that there is a risk of injury when dissecting over the knee. The patient can experience medial leg numbness or dysesthesia from its injury.

The recto-vaginal fistula was completely excised, resulting in a larger passage. The gracilis muscle had sufficient bulk to plug the entire defect. It was loosely tagged down to avoid strangulation, relying mainly on the pull-through tendon for anchorage. The recurrence doubtless took place as a result of multiple surgical attempts in a scarred operative field; hence, radical excision and well-vascularized tissue were necessary for complete healing.

Another flap option is the Singapore flap, which is a fasciocutaneous flap supplied by the posterior labial branches of the pudendal artery [12]. The flap can be de-epithelized and interposed between the vagina and rectum [13]. The donor site scar is hidden in the inner thigh crease. We believe that this flap is an option in cases where the operative field is less scarred. In our case, we preferred a muscle flap because it has better vascularity.

We believe in this case, fecal diversion alone was inadequate for primary healing, despite being advocated by some authors [14]. Prior to the present reconstructive attempt, a digital rectal examination revealed mucus plugs and contact bleeding, sug- 
gesting chronic inflammation. A stoma was created 1 month before surgery to improve hygiene. The stoma was reversed 6 months later, after endoscopic examination confirmed healing. However, 1 week after stoma reversal, the patient reported flatus passage and staining in the vagina. She also strained excessively during defecation owing to spasm of the anal sphincter after months of disuse. We postulated that the high voiding rectal pressure caused a temporary dehiscence of the repair. However, proctoscopy revealed no visible tract and a vaginal examination showed no fecal content. The patient was immediately advised to stop straining and to employ manual assistance for complete bowel evacuation. During this period, she underwent physicianassisted biofeedback every other day. This was accomplished by asking the patient to simulate voiding during rectal digitation, with the physician providing tactile and verbal feedback to the patient on how tense or relaxed the anal sphincter was. As this process was repeated, the patient improved the coordination and relaxation of the anal sphincter. The patient reported improvement of symptoms after 1 month.

For future cases, biofeedback should be commenced before and after reversal of the stoma to ensure smooth passage of stool. Adjunctive measures such as use of polyethylene glycol and mineral oil enemas can aid in stool softening and lubrication. Since successful repair can only be established after stoma reversal and resumption of normal fecal passage, it behooves the reconstructive surgeon to closely monitor the patient after stoma reversal.

\section{NOTES}

\section{Conflict of interest}

No potential conflict of interest relevant to this article was reported.

\section{Ethical approval}

The study was performed in accordance with the principles of the Declaration of Helsinki. Written informed consent was obtained.

\section{Patient consent}

The patient provided written informed consent for the publication and the use of her images.

\section{Author contribution}

Case acquisition, analysis and interpretation, drafting of manuscript: Mok WL. Study concept and design, critical review of manuscript, study supervision: Tan BK. Case acquisition, analysis and interpretation: Goh MH. Study concept and design: Tang CL.

\section{ORCID}

Wan Loong James Mok https://orcid.org/0000-0001-94761318

Ming Hui Goh https://orcid.org/0000-0001-6342-4001

Choong Leong Tang https://orcid.org/0000-0003-4310-6588

Bien Keem Tan https://orcid.org/0000-0002-7347-8713

\section{REFERENCES}

1. Senatore PJ Jr. Anovaginal fistulae. Surg Clin North Am 1994;74:1361-75.

2. Corte H, Maggiori L, Treton X, et al. Rectovaginal fistula: what is the optimal strategy? An analysis of 79 patients undergoing 286 procedures. Ann Surg 2015;262:855-60.

3. Champagne BJ, O'Connor LM, Ferguson M, et al. Efficacy of anal fistula plug in closure of cryptoglandular fistulas: long-term follow-up. Dis Colon Rectum 2006;49:1817-21.

4. Grimaud JC, Munoz-Bongrand N, Siproudhis L, et al. Fibrin glue is effective healing perianal fistulas in patients with Crohn's disease. Gastroenterology 2010;138:2275-81.

5. McNevin MS, Lee PY, Bax TW. Martius flap: an adjunct for repair of complex, low rectovaginal fistula. Am J Surg 2007; 193:597-9.

6. Lefevre JH, Bretagnol F, Maggiori L, et al. Operative results and quality of life after gracilis muscle transposition for recurrent rectovaginal fistula. Dis Colon Rectum 2009;52: 1290-5.

7. Gottgens KW, Heemskerk J, van Gemert W, et al. Rectovaginal fistula: a new technique and preliminary results using collagen matrix biomesh. Tech Coloproctol 2014;18:81723.

8. Mukwege D, Mukanire N, Himpens J, et al. Minimally invasive treatment of traumatic high rectovaginal fistulas. Surg Endosc 2016;30:379-87.

9. Gajsek U, McArthur DR, Sagar PM. Long-term efficacy of the button fistula plug in the treatment of Ileal pouch-vaginal and Crohn's-related rectovaginal fistulas. Dis Colon Rectum 2011;54:999-1002.

10. Furst A, Schmidbauer C, Swol-Ben J, et al. Gracilis transposition for repair of recurrent anovaginal and rectovaginal fistulas in Crohn's disease. Int J Colorectal Dis 2008;23:349-53.

11. Papadopoulos O, Konofaos P, Georgiou P, et al. Gracilis myocutaneous flap: evaluation of potential risk factors and long-term donor-site morbidity. Microsurgery 2011;31: 448-53.

12. Wee JT, Joseph VT. A new technique of vaginal reconstruction using neurovascular pudendal-thigh flaps: a preliminary report. Plast Reconstr Surg 1989;83:701-9. 
13. Levinson H (Duke University, USA). Duke flap course. Conversation with: Tan BK (Singapore General Hospital, Singapore). 2016 Aug 5.
14. Regimbeau JM, Panis Y, Cazaban L, et al. Long-term results of faecal diversion for refractory perianal Crohn's disease. Colorectal Dis 2001;3:232-7. 